\title{
Two Antiphase Oscillations Occur in Each Suprachiasmatic Nucleus of Behaviorally Split Hamsters
}

\author{
Lily Yan, ${ }^{1}$ Nicholas C. Foley, ${ }^{1}$ Jessica M. Bobula, ${ }^{1}$ Lance J. Kriegsfeld, ${ }^{1}$ and Rae Silver ${ }^{1,2,3}$ \\ Departments of ${ }^{1}$ Psychology and ${ }^{2}$ Anatomy and Cell Biology, Columbia University, and ${ }^{3}$ Department of Psychology, Barnard College of Columbia \\ University, New York, New York 10027
}

\begin{abstract}
The suprachiasmatic nuclei (SCNs) control circadian rhythms of numerous behavioral and physiological responses. In hamsters, constant light causes "splitting" of circadian rhythms, such that a single daily bout of activity separates into two components, $12 \mathrm{~h}$ apart, with antiphase circadian oscillations in the left and right SCN. Given the phenotypic and functional heterogeneity of the SCN, in which ventrolateral but not dorsomedial neurons are retinorecipient, we asked how these two compartments respond to the constant lighting conditions that produce splitting, using three different phase markers of neuronal activity: PER1 (Period 1), c-FOS, and pERK (phosphorylated extracellular signal-regulated kinase). We report the emergence of a coherent novel network in which each side of the SCN exhibits two antiphase oscillating subregions, here termed "core-like" and "shell-like," in addition to the known antiphase oscillation between the right and left SCN. The novel SCN response entails a coherent rhythm in a core-like region of the SCN, which otherwise is not cycling. A mathematical model is presented, and this model interprets the observed changes in the proportion of in-phase and antiphase populations of SCN oscillators and suggests novel testable hypotheses. Finally, the functional significance of this network was explored by investigating the adjacent hypothalamus. Activation of the paraventricular nucleus is in-phase with the ipsilateral core-like SCN, whereas activation of the lateral subparaventricular zone is in-phase with the ipsilateral shell-like SCN, pointing to a multiplicity of SCN output signals. These results suggest a neural basis for internal coincidence of SCN oscillators, and a novel mechanism of plasticity in SCN neural networks and outputs.
\end{abstract}

Key words: circadian rhythms; behavioral splitting; SCN; PER1; SCN efferents; oscillator model

\section{Introduction}

Daily rhythms in behavioral and physiological responses are controlled by a network of pacemakers located in the suprachiasmatic nuclei (SCNs) for mammals (Klein et al., 1991). The SCN is peptidergically heterogeneous, with $\sim 10,000$ neurons on each side of the brain, and has functionally distinct regions with regard to electrical activity and clock gene expression (Antle and Silver, 2005). Circadian rhythmicity is a property of individual SCN neurons, and the molecular machinery within each cell is comprised of a transcription/translation-based feedback loop, in which the expression of "clock genes" is suppressed periodically by their protein products (Welsh et al., 1995; Reppert and Weaver, 2001). Whereas the SCN as a whole produces a coherent rhythm regulating responses in the rest of the body, individual SCN neurons within the nucleus maintain diverse circadian periods and phases (Quintero et al., 2003; Yamaguchi et al., 2003). Coupling among individual neurons can be identified in regional differences of the SCN. In hamsters, neurons in the ventrocaudal

Received June 20, 2005; revised July 25, 2005; accepted Aug. 15, 2005.

This work was supported by National Institute of Neurological Disorders and Stroke Grant 37919 to R.S. We thank Dr. Michael Lehman for the provision of PER1 antibody, and Drs. Duncan Foley, Michael Antle, and Joseph LeSauter for helpful comments on a previous draft of this manuscript.

Correspondence should be addressed to Dr. Rae Silver, Department of Psychology, Columbia University, 406 Schermerhorn Hall, MC5501, 1190 Amsterdam Avenue, New York, NY 10027. E-mail: qr@columbia.edu.

DOI:10.1523/JNEUROSCI.2538-05.2005

Copyright $\odot 2005$ Society for Neuroscience $\quad$ 0270-6474/05/259017-10\$15.00/0 (core) SCN receive direct retinal input via the retinohypothalamic tract (RHT) and lack detectable rhythms in clock gene expression and electrical activity (Bryant et al., 2000; Hamada et al., 2001; Jobst and Allen, 2002). In contrast, the dorsal (shell) SCN contains cells expressing robust circadian rhythmicity in clock genes and clock-controlled genes, although mostly lacking direct retinal input. More surprisingly, a small population of cells exhibit rhythmic phosphorylated extracellular signal-regulated kinase (pERK), which is dependent on the eye and cycles in antiphase to the pERK rhythm in the shell SCN (Lee et al., 2003a). These studies point to an important role for retinal input and photic cues in contributing to SCN function.

Much attention has been directed at the effects of acute light pulses and daily transitions between dawn and dusk as entraining signals. Tonic effects of light have received far less attention, although it is known that photic history and light intensity affect circadian rhythms in humans and nonhuman animals (Shimomura et al., 1997; Sudo et al., 2003; Smith et al., 2004). Given that RHT innervation is mostly restricted to neurons in the ventrolateral, core SCN (Moore, 1973; Bryant et al., 2000; Abrahamson and Moore, 2001), we asked how the core and shell SCN each respond to tonic light exposure. To this end, we examined the SCN of hamsters housed in constant light (LL). Housing in LL can produce behavioral "splitting," in which the animal's single daily bout of activity separates into two components, each component free run until they become stably coupled $\sim 12 \mathrm{~h}$ apart 


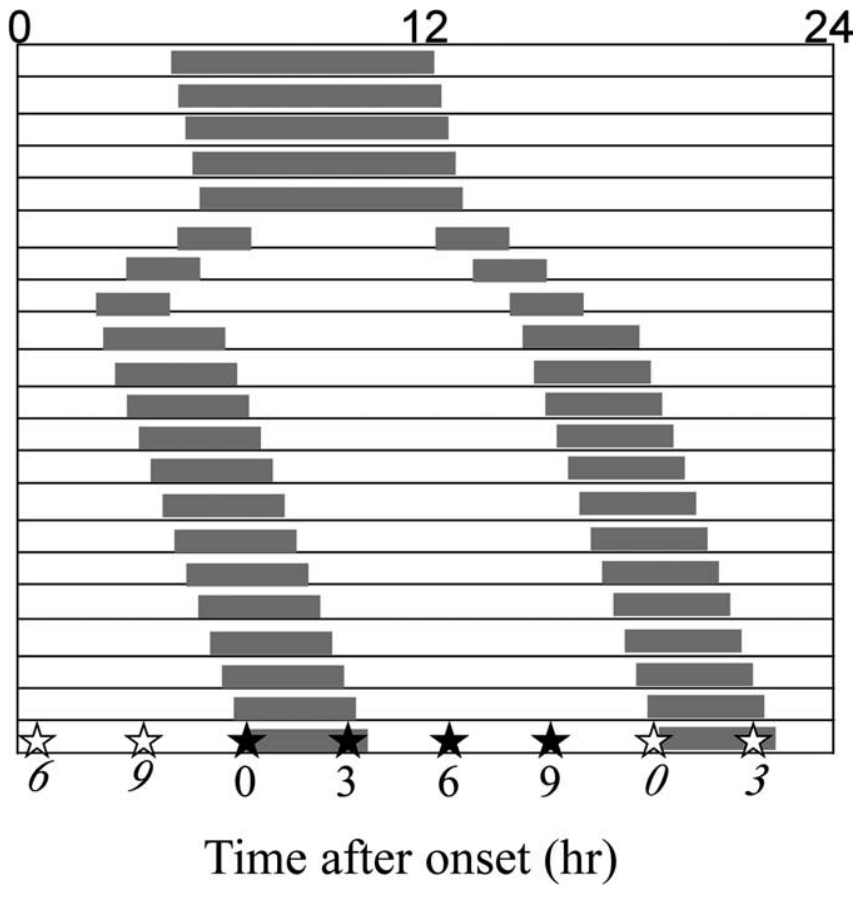

Figure 1. Experimental design. When hamsters showed stable splitting, they were killed every $3 \mathrm{~h}$ after the onset of one (open stars) or the other (closed stars) activity bout. Unsplit controls were killed at the corresponding circadian times.

(Pittendrigh and Daan, 1976). This is thought to be the result of antiphase oscillation of clock genes between the right and left side of the SCN (de la Iglesia et al., 2000). In the present study, we compared the protein product of Period 1 (PER1) in the core and shell SCN in control and split hamsters to analyze the unique response of each region. To assess the relationship between the brain clock and hypothalamic targets of SCN efferents, we also examined c-FOS and pERK activity.

\section{Materials and Methods}

Animals. Male Syrian hamsters $(n=50$; Charles River Laboratories, Kingston, NY), 4 weeks of age at the time of purchase, were housed individually in constant light (LL) (800 lux; $n=44)$ or constant dark (DD) $(n=6)$ in cages equipped with running wheels (diameter, $16 \mathrm{~cm})$. Food and water were available ad libitum. The room was equipped with a white-noise generator (91 dB sound pressure level) to mask environmental noise. For animals housed in DD, a dim red light ( $<1$ lux; Delta 1, Dallas, TX) allowed for husbandry and manipulations. Wheel-running activity was monitored continuously via a computer-based data acquisition system (Dataquest; Data Sciences, St. Paul, MN) that summed wheel revolution counts into $10 \mathrm{~min}$ bins. Data was downloaded and analyzed with Circadia (Behavioral Cybernetics, Cambridge, MA). Studies were conducted in accordance with the regulations of the Columbia University Institutional Animal Care and Use Committee.

Splitting of wheel-running activity occurred after 8 weeks of exposure to LL, as in previous reports (de la Iglesia et al., 2000). After 2 weeks of stable splitting behavior, hamsters were killed at $0,3,6$, or $9 \mathrm{~h}$ after either one of the activity onsets, shown schematically in Figure 1. Unsplit controls, which retained a single coherent bout of activity after at least 8 weeks in LL, were killed every $3 \mathrm{~h}$ throughout the circadian cycle. Another group of control animals were housed in DD for 2 weeks and killed at circadian time 0 (CT0) and CT12.

Immunocytochemistry. Animals were anesthetized (pentobarbital; 200 $\mathrm{mg} / \mathrm{kg}$, i.p.) and perfused intracardially with saline followed by fixative (4\% paraformaldehyde in $0.1 \mathrm{~m}$ phosphate buffer). Brains were removed and postfixed overnight in the same fixative, and then cryoprotected in $20 \%$ sucrose for $2 \mathrm{~d}$. Brains were marked with a cut to identify the left and right sides. Alternate coronal sections $(40 \mu \mathrm{m})$ were prepared using a cryostat.

Triple-label immunocytochemistry for PER1 or c-FOS with vasopressin and calbindin ${ }_{D 28 K}$. Split hamsters $(n=3-4 /$ time point $), \mathrm{LL}$ and DD controls ( $n=2-3 /$ time point) were processed as follows. The first label was either PER1 (raised in goat; 1:4000; gift of Dr. M. Lehman, University of Cincinnati, Cincinnati, OH), or c-FOS (raised in rabbit; 1:10,000; Santa Cruz Biotechnology, Santa Cruz, CA); the second and third labels were calbindin $_{\mathrm{D} 28 \mathrm{~K}}(\mathrm{CalB})$ (raised in mouse; 1:10,000; Sigma, St. Louis, MO) and arginine vasopressin (AVP) (raised in guinea pig; 1:10,000; Peninsula Laboratories, San Carlos, CA). The specificity of PER1 antibody has been characterized previously and was confirmed by preadsorption with the immunogen peptide (M. Lehman, personal communication).

Immunocytochemistry (ICC) for PER1 or c-FOS with CalB and AVP on free-floating sections started with a $1 \mathrm{~h}$ blocking incubation in $2 \%$ normal donkey serum (NDS) in phosphate buffer with $0.1 \%$ Triton $\mathrm{X}-100(0.1 \% \mathrm{PBT})$, followed by $48 \mathrm{~h}$ incubation in the primary antibody (in $0.1 \%$ PBT plus NDS) at $4^{\circ} \mathrm{C}$. Sections were rinsed three times in $0.1 \mathrm{M}$ $\mathrm{PBT}$, and then incubated in the following fluorescent secondary antibodies: cyanine 3 (Cy3)-donkey-anti-goat or Cy3-donkey-anti-rabbit, Cy2donkey-anti-guinea pig, and Cy5-donkey-anti-mouse (each at 1:200; Jackson ImmunoResearch, West Grove, PA) for $2 \mathrm{~h}$ at room temperature, and then rinsed three times in $0.1 \mathrm{M}$ PBT. After the ICC reaction, sections were mounted on gelatin-coated slides, dehydrated with alcohol rinses, cleared with xylene, air dried, and coverslipped with Krystalon. In all cases, the tissue was protected from light exposure once a fluorescent secondary was applied.

Triple-label ICC for PER1, pERK, and CalB. Split hamsters killed 3 and $9 \mathrm{~h}$ after activity onset time $(n=2)$ were used for this preparation. For pERK (raised in rabbit; 1:400; Cell Signaling Technology, Beverly, MA) ICC, an amplification protocol was used (Antle et al., 2005). Briefly, the tissue was incubated in pERK antibody for $48 \mathrm{~h}$ at $4^{\circ} \mathrm{C}$ followed by rinses in PBT (three times), incubation in biotinylated goat anti-rabbit secondary antibody (Vector Laboratories, Burlingame, CA), rinses in PBT (three times), and incubation in $0.2 \%$ avidin-biotin complex (Vectastain Elite ABC; Vector Laboratories). After PBT rinses (three times), the tissue was incubated for $30 \mathrm{~min}$ in biotinylated tyramide with $0.1 \%$ hydrogen peroxide in $0.1 \mathrm{M}$ PBS, and then rinsed in PBS (three times), incubated for $1 \mathrm{~h}$ in Cy2-conjugated egg-white avidin (1:200; Jackson ImmunoResearch), and again rinsed in PBT (five times) and then immunoreacted for PER1 and CalB as above.

Delineation of distinct SCN regions. A number of authors have attempted to optimize the terminology used to describe subregions of the SCN. Initial studies of the rat distinguished between the ventrolateral and dorsomedial SCN (van den Pol and Tsujimoto, 1985). Because the "geographical" designations did not apply uniformly across species, this was subsequently replaced with the more general terms "core" and "shell"(Moore, 1996).

In this paper, we use the terms "core-like" and "shell-like" to delineate the SCN region showing in-phase and antiphase rhythms of circadian oscillation, because neither of the conventional terminologies applies in full. The term "core-like" includes the CalB-expressing cells lying in the third quadrant of the rostrocaudal SCN (Silver et al., 1996a; Hamada et al., 2001) and the region of retina-dependent antiphase pERKimmunoreactive (IR) cells lying dorsal to the CalB cells (Lee et al., 2003a,b). The term "shell-like" describes the remainder of the SCN.

Image analysis. Images were captured using a Nikon Eclipse E800 microscope (Nikon, Tokyo, Japan) equipped with the following filters: GFP (green fluorescent protein), TX Red (Texas Red), and Cy5, which specifically pass the signal for $\mathrm{Cy} 2, \mathrm{Cy} 3$, and $\mathrm{Cy} 5$, respectively. The microscope is fitted with a cooled CCD digital camera with SPOT software (Diagnostic Instruments, Sterling Heights, MI). Images were loaded into Photoshop 7.0 (Adobe Systems, San Jose, CA) for cell counting. PER1-positive cells were counted bilaterally in the core-like and the shell-like regions of the SCN. The magnitude of asymmetry was presented as the left-right ratio of the PER1-positive cells in corresponding regions of the split SCN.

Confocal microscopy was used to examine the colocalization of PER1, c-FOS, or pERK with CalB using Zeiss LSM 510 (Zeiss, Thornwood, NY). 
A

\section{rostral SCN}

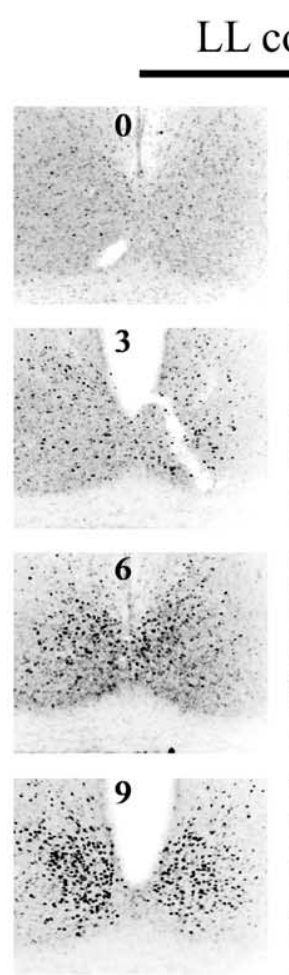

B

\section{caudal SCN}

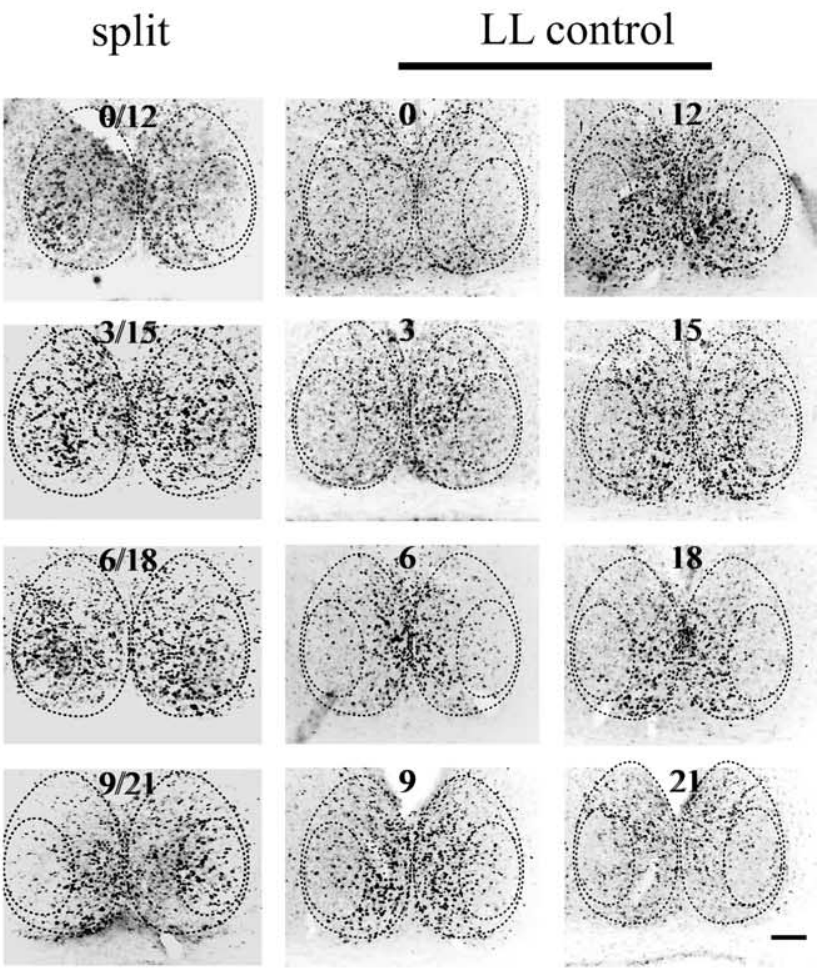

Figure 2. PER1 staining in the rostral and caudal portion of the SCN in the split and unsplit control hamsters. Images of rostral $(\boldsymbol{A})$ and corresponding caudal $(\boldsymbol{B}) \mathrm{SCN}$ are from the same animal. The circadian time sampled is indicated in each image. Dashed lines indicate the core- and shell-like SCN regions, based on CalB and VP staining. The rostral $(\boldsymbol{A})$ and caudal $(\boldsymbol{B})$ SCN sections from split animals are arranged side by side to facilitate comparison. Scale bar, $100 \mu \mathrm{m}$.

Sections were excited sequentially with an argon-krypton laser using the standard excitation wavelength for Cy2, Cy3, and Cy5. Stacked images were collected as $1 \mu \mathrm{m}$ multitract optical sections. LSM 3.95 software (Zeiss) was used to superimpose images of the sections.

Mathematical model. The mathematical model was created in Mathematica 5.1 (Wolfram Research, Champaign, IL), on an Apple PowerBook G4 (Apple Computer, Cupertino, CA), and is an extension of the notebook developed in Antle et al. (2003).

Statistical analysis. Data were analyzed with unpaired $t$ tests or Kruskal-Wallis analysis, as appropriate. All results are reported as means \pm SEM.

\section{Results \\ Antiphase rhythmic PER1 expression in the core-like region of the split SCN}

PER1 expression was examined through the entire extent of the $\mathrm{SCN}$ in animals killed at $3 \mathrm{~h}$ intervals throughout the day. Figure 2 presents micrographs summarizing the results, with rostral $(A)$ and caudal $(B)$ sections for each time point taken from the same individual. In the rostral aspect of the shell-like region (Fig. 2A), PER1 staining in LL controls was high at CT9, CT12, CT15, and CT18; moderate at CT6; and low at CT0, СT3, and CT21. By aligning the micrographs from the control and split animals, it is clear that PER1 was asymmetric in the behaviorally split animal at all time points examined, with the right and left SCN oscillating in antiphase as previously reported (de la Iglesia et al., 2000). Note that the caudal SCN encompasses both core- and shell-like subregions (Fig. $2 \mathrm{~B}$ ), and PER1 expression in the shell-like area of the caudal SCN is similar to that of the rostral SCN. In behaviorally split animals, the shell-like SCN had the same pattern of
PER1 expression as in control unsplit animals, although the SCN on one side resembled subjective day and the other side subjective night.

The novel result is seen in the core-like SCN of split animals, with rhythmic PER1 in the core-like region in antiphase to its expression in the ipsilateral shell-like region (Fig. $2 B$, left panel). That is, the PER1 rhythms in the core- and shell-like region of the split animal were in antiphase, within each SCN. As seen in Figure $2 B$, right panel, there was no detectable rhythm of PER1 in the core-like region of LL control animals, although individual, scattered PER1-expressing cells were seen.

Confocal microscopy of triple-labeled sections and cell counting were used to further compare the SCN at the level of the CalB area in split (CT12/0) and control animals at CT12 and CT0 (Fig. $3 A$ ). In the shell-like region delineated by vasopressin (VP) cells, there was a clear left/right asymmetry of PER1 expression in the split SCN, with one side resembling CT12 and the other CT0 of unsplit animals (Fig. $3 A$ ). In the CalB-containing core-like region, PER1 expression was low in controls at both CT0 and CT12, and high in the split SCN on the side bearing low shell-like expression. These observations were quantified by counting PER1containing cells in split and control animals, housed in DD or LL (Fig. 3B). In the SCN PER1 expression is high at CT12, the time of activity onset, and low at CT0 (Field et al., 2000; NuessleinHildesheim et al. 2000; Sumova et al., 2002; Yan and Silver, 2004; present study). This information was used as follows: for split animals killed at the onset of activity (CT12/0), the side of the SCN with high PER1 immunoreactivity in the shell region (cor- 
responding to the controls at CT12) was defined as the "CT12 side." The other side of the SCN was defined as the "CT0 side." In the shell-like area, the number of PER1 cells was high at CT12 and low at CT0 in all three groups. In the core-like region, there were no differences between CT0 and CT12 in the LL and DD unsplit control groups, with PER1 expression low at both time points. In the split SCN, however, the number of PER1 cells in the corelike region was much higher on the CT0 side than that on the CT12 side $(p<0.01$; unpaired $t$ test). The asymmetry in the core- and shell-like regions of the split $\mathrm{SCN}$ at $3 \mathrm{~h}$ intervals through the circadian cycle is shown in Figure $3 C$. The results indicate that when the left-right ratio of PER1 cell numbers in the shell-like area was at its peak $(p<0.05$; Kruskal-Wallis analysis), the ratio in the core-like area was at its nadir $(p<0.05$; Kruskal-Wallis analysis) and vice versa.

It is noteworthy that, although the antiphase PER1 expression occurred in the region delineated by CalB cells, these proteins were not colocalized. There was no difference in the fraction of CalB cells expressing PER1 on the two sides of the corelike SCN (high side, $7.2 \pm 1.4 \%$; low side, $6.3 \pm 1.9 \% ; t$ test; $p>0.05 ; n=4$ animals).

The antiphase rhythms within each SCN were confirmed with two additional phase markers, c-FOS (Fig. 4, left panel) and pERK (see Fig. 6A). Although DDhoused animals express $\mathrm{pERK}$ in a core area localized dorsal to CalB cells (Lee et al., 2003b; Antle et al., 2005), we found that, in split hamsters, antiphase pERK was expressed in a much larger area lying dorsal to, overlapping with, and ventral to CalB cells (see Fig. $6 \mathrm{~A}$, left panel). This was confirmed quantitatively: in split hamsters, the area of antiphase pERK expression was $\sim 45,000 \mu \mathrm{m}^{2}$, much larger than the $\sim 23,000 \mu \mathrm{m}^{2}$ in the controls.

c-FOS, pERK, and PER1 were all rhythmic, but they were not simultaneously expressed, consistent with previous reports (Chambille et al., 1993; Guido et al., 1999; Field et al., 2000; Lee et al., 2003a). At $3 \mathrm{~h}$ after activity onset, c-FOS was high on the side contralateral to high PER1, whereas at $9 \mathrm{~h}$ after activity onset, it was high on the same side as PER1 for both core- and shell-like areas.

Three hamsters that did not show behavioral splitting nevertheless exhibited left-right asymmetry of PER1 and c-FOS expression in the SCN. This finding suggests that the SCN splits before behavioral splitting and agrees with a previous report by de la Iglesia et al. (2003).

PVN activity is correlated with ipsilateral core-like SCN; lateral subparaventricular zone activity is correlated with ipsilateral shell-like SCN

We next assessed the temporal relationship of neuronal activity in the SCN to that of adjacent hypothalamic nuclei, using c-FOS (Figs. 4, 5) and pERK (Fig. 6). CalB immunoreactivity was used to ensure that the hypothalamic section being examined was bilaterally symmetrical. Figure 4 shows micrographs aligned from a representative split animal killed $3 \mathrm{~h}$ after activity onset, with the SCN in the left panels, the PVN in the middle panels, and the lateral subparaventricular zone (LSPVZ) in the right panels. In the SCN, high c-FOS activity occurred in the shell-like region of the right SCN and core-like region of the left side (Fig. 4, left panels). In the PVN, high c-FOS expression was found in the left PVN localized to VP-expressing magnocellular neurons and other unidentified cells (Fig. 4, middle panels). In LSPVZ, c-FOS was also bilaterally asymmetrical, with higher expression ipsilateral to the high c-FOS expression in the shell-like area (Fig. 4, right panels). The same results were observed in all animals killed at this time point. c-FOS expression is high during the day and low during the night in the SCN shell (Chambille et al., 1993; present study). This was used to assign the right and left SCN of the split animal to its appropriate time of day of either CT3 or CT15. The right-left ratio was calculated for each region (shelllike SCN, $5.13 \pm 0.34 ;$ LSPVZ, $5.83 \pm 0.43$; core-like SCN, $0.21 \pm$ 0.02 ; PVN, $0.28 \pm 0.01 ; n=3$ ). Similar in-phase activation of shell-like SCN and LSPVZ was seen in the control animals killed at CT3 and CT15, respectively (Fig. 5). c-FOS activity was high in 


\section{CT3/15}
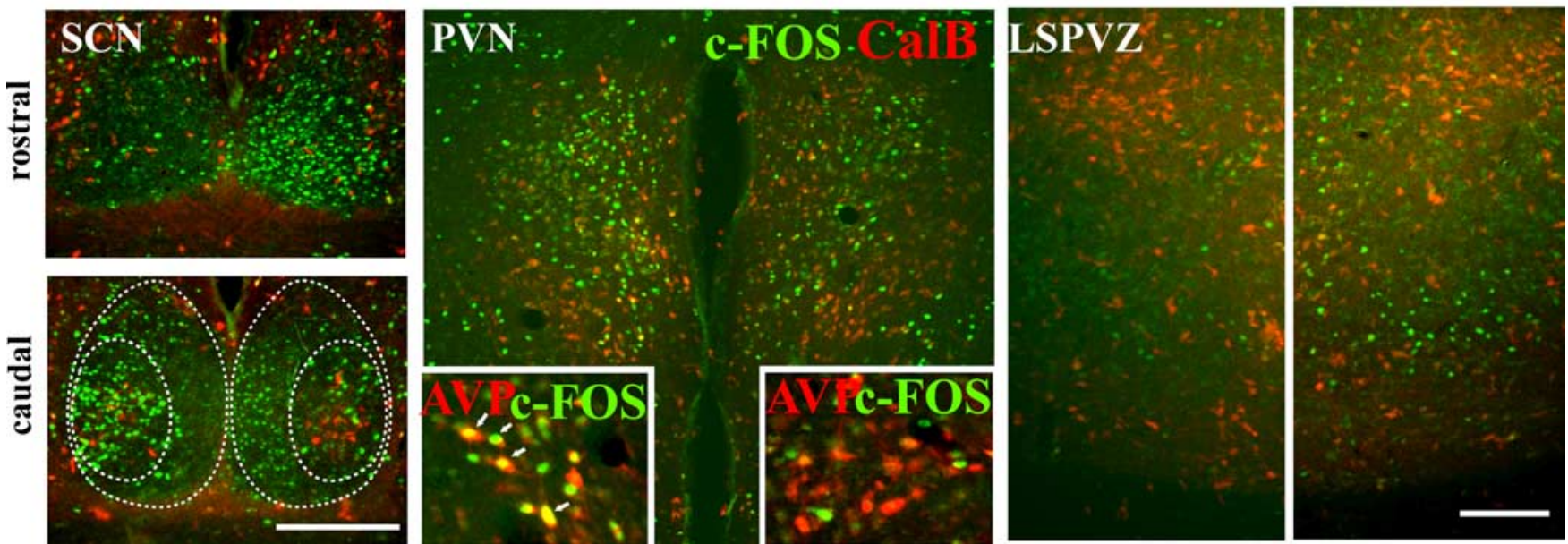

Figure 4. Double label of C-FOS/CaIB in the SCN, PVN, and LSPVZ of a representative split animal killed at $3 \mathrm{~h}$ after activity onset (CT3/15). The CalB label was used as a marker for the core-like SCN and to show the symmetry of the hypothalamic sections. High c-FOS expression in PVN is on the same side as in the core-like SCN, whereas high c-FOS in LSPVZ is on the same side as in the shell-like SCN. Scale bars, $300 \mu \mathrm{m}$.

A
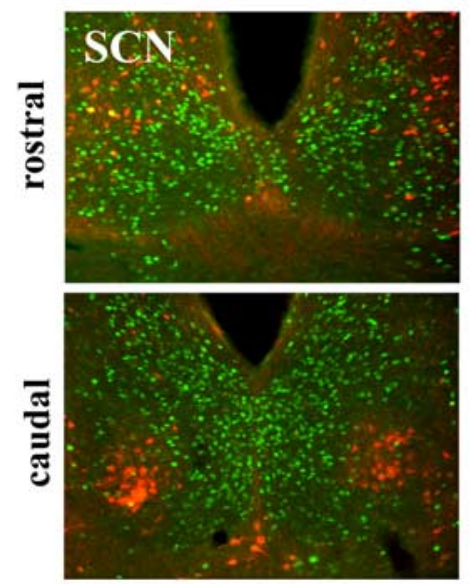

B
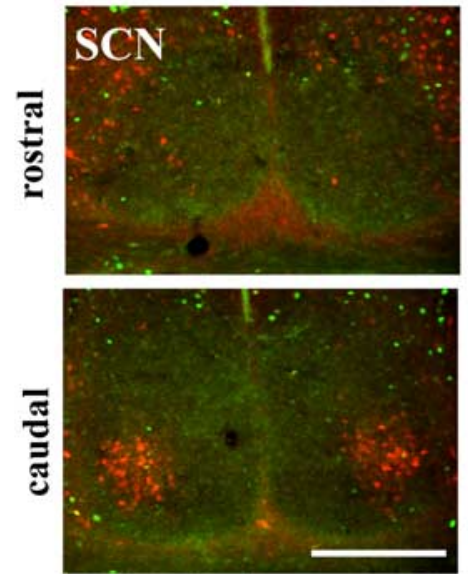

CT3
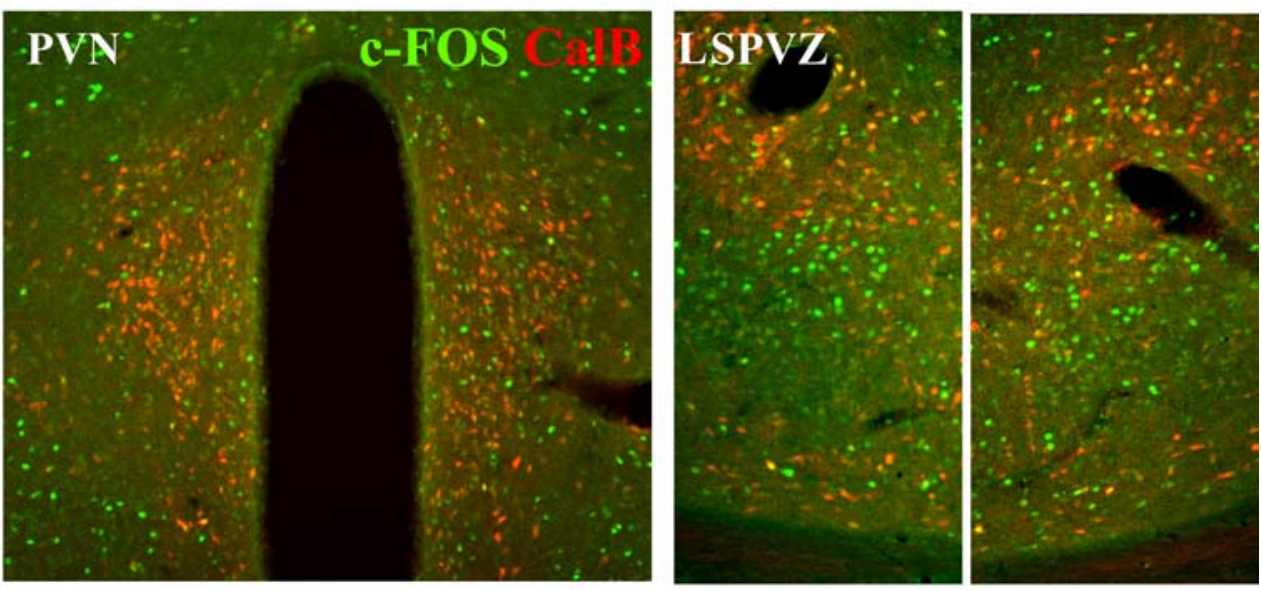

\section{CT15}

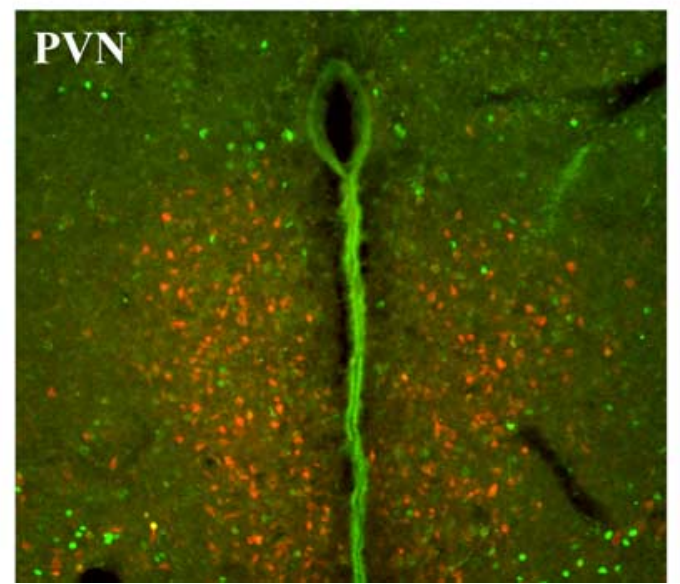

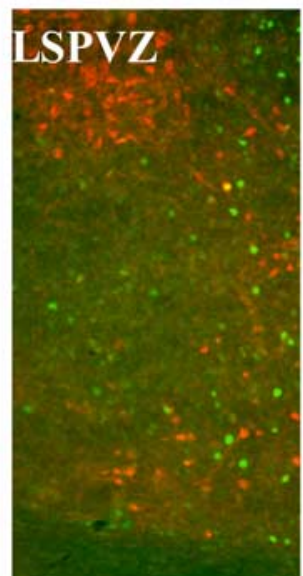

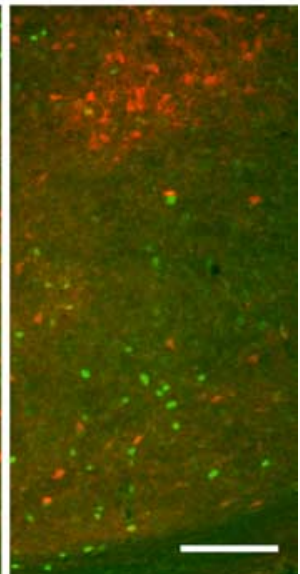

Figure 5. Double label of C-FOS/CaIB in the SCN, PVN, and LSPVZ of two unsplit control animals killed at CT3 $(\boldsymbol{A})$ and $C T 15(\boldsymbol{B})$. The CalB label was used as a marker for the core-like SCN and to show the symmetry of the hypothalamic sections. Scale bars, $300 \mu \mathrm{m}$. 
A
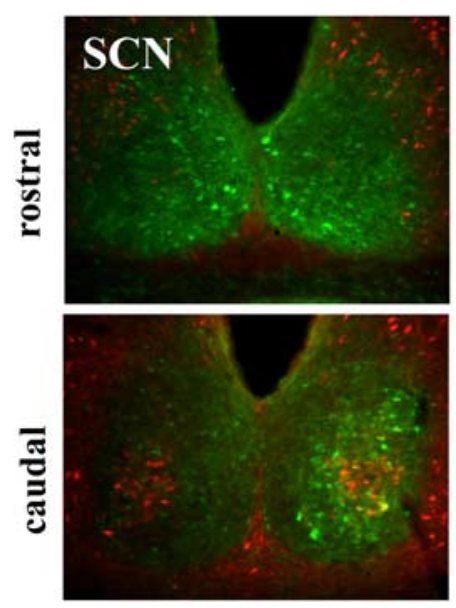

B
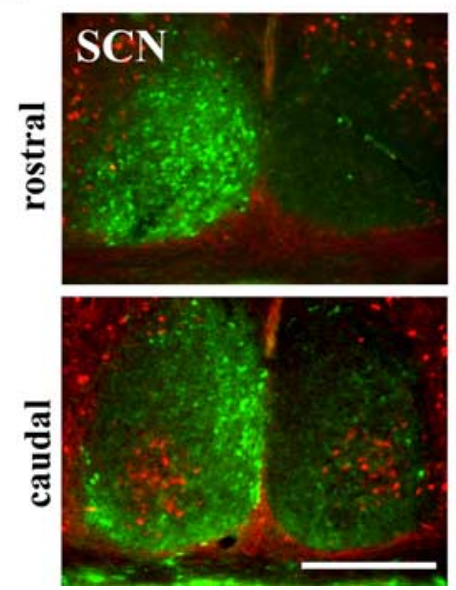

CT3/15
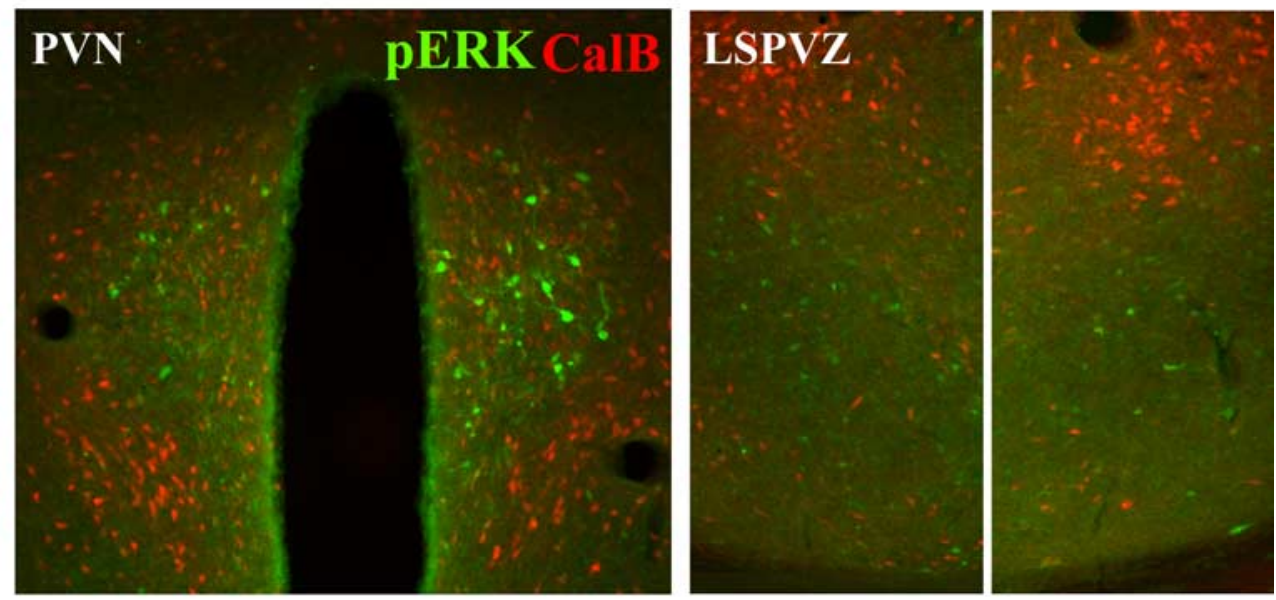

CT9/21

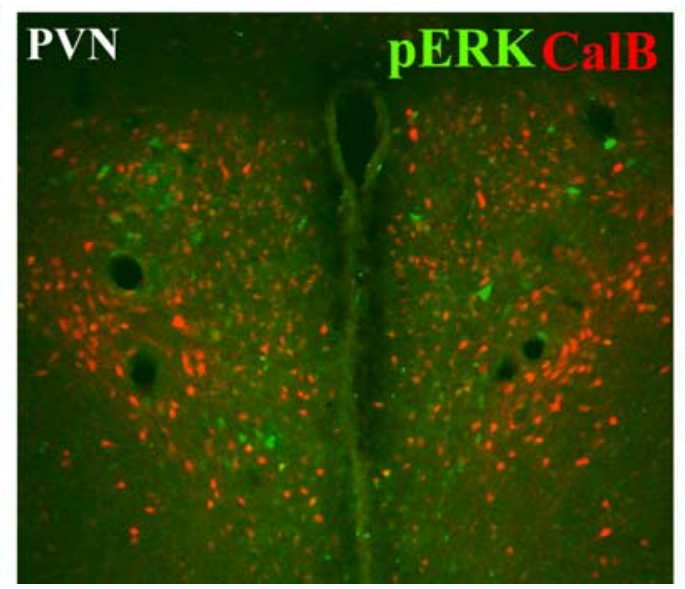

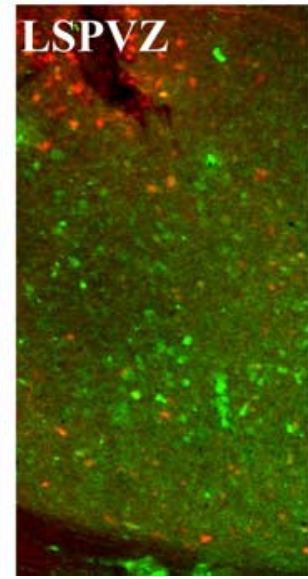

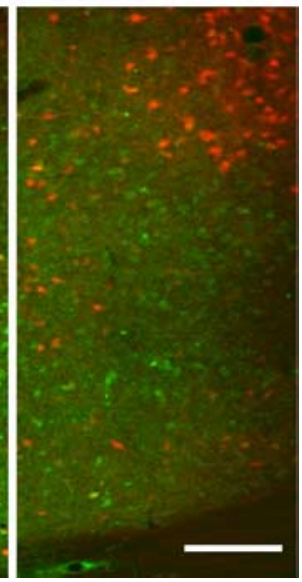

Figure 6. Double label of pERK/CaIB in the SCN, PVN, and LSPVZ of two representative split animals from $3 \mathrm{~h}(\boldsymbol{A}$; $(\mathrm{CT} 3 / 15)$ and $9 \mathrm{~h}(\boldsymbol{B}$; $(\mathrm{CT} 9 / 21)$ after activity onset. The CaIB label was used as a marker for the core-like SCN and to show the symmetry of the hypothalamic sections. High pERK expression in PVN is on the same side as it is in the core-like SCN, whereas high pERK in LSPVZ is on same side as it is in the shell-like SCN. Scale bars, $300 \mu \mathrm{m}$.

the shell-like SCN at CT3, but low at CT15. The same pattern was seen in LSPVZ, with c-FOS being high at CT3 and low at CT15. However, there was little c-FOS activity in controls for either the core-like SCN or the PVN at either time point.

The foregoing results were confirmed using pERK. Figure 6 shows micrographs of two representative split hamsters killed $3 \mathrm{~h}$ (Fig. 6A) or $9 \mathrm{~h}$ (Fig. $6 \mathrm{~B}$ ) after activity onset, aligned as in Figure 4 . At $3 \mathrm{~h}$ after activity onset, pERK is bilaterally asymmetrical and very high on the right side of core-like SCN, with low to moderate bilaterally symmetrical expression in the shell-like SCN (Fig. 6A, left panels). In the PVN, pERK staining is unilateral and high on the right side (Fig. $6 A$, middle panel). In the LSPVZ, pERK is low on both sides (Fig. $6 A$, right panel). In this animal, the high pERK expression in the PVN and the core-like area in the SCN occurred on the same side. At $9 \mathrm{~h}$ after activity onset, $\mathrm{pERK}$ was high on the left side of the shell-like area, but symmetrically low to moderate on both sides of the core-like SCN (Fig. 6B, left panels). In the PVN, pERK was low on both sides (Fig. 6B, middle panel). In the LSPVZ, pERK was asymmetric and high on the left side, the same as the shell-like SCN.

In summary, the results indicate that, in the split animal, high c-FOS and pERK activity occur at corresponding times in the ipsilateral PVN and core-like SCN, and are antiphase to the ipsi- lateral LSPVZ and shell-like SCN. In other hypothalamic regions, no obvious asymmetry was noted, although detailed cell counting or identification of specific nuclei will likely reveal additional differences. In the lateral habenula, very strong unilateral expression of c-FOS was noted in three of six animals examined at CT3/15 and СT9/21 but in none of the animals examined at CT0/12 and CT6/18 $(n=6)$.

\section{Mathematical model of in-phase and antiphase cells}

To explore the emergence of in-phase and antiphase populations of cells, we extended our previous gate-oscillator model (Antle et al., 2003), which incorporates several known properties of SCN neurons including nonoscillating gate cells that are directly retinorecipient and a large population of oscillator cells. The model oscillators follow a polar van der Pol equation:

$$
\begin{aligned}
& \frac{d r}{d t}=-\varepsilon \omega r \cos (\theta)^{2}\left[-1+r^{2} \cos (\theta)^{2}\right] \\
& \frac{d \theta}{d t}=\frac{\omega}{2}\left[-2+2 \varepsilon r^{2} \cos (\theta)^{3} r^{2} \sin (\theta)-\varepsilon \sin (2 \theta)\right]
\end{aligned}
$$



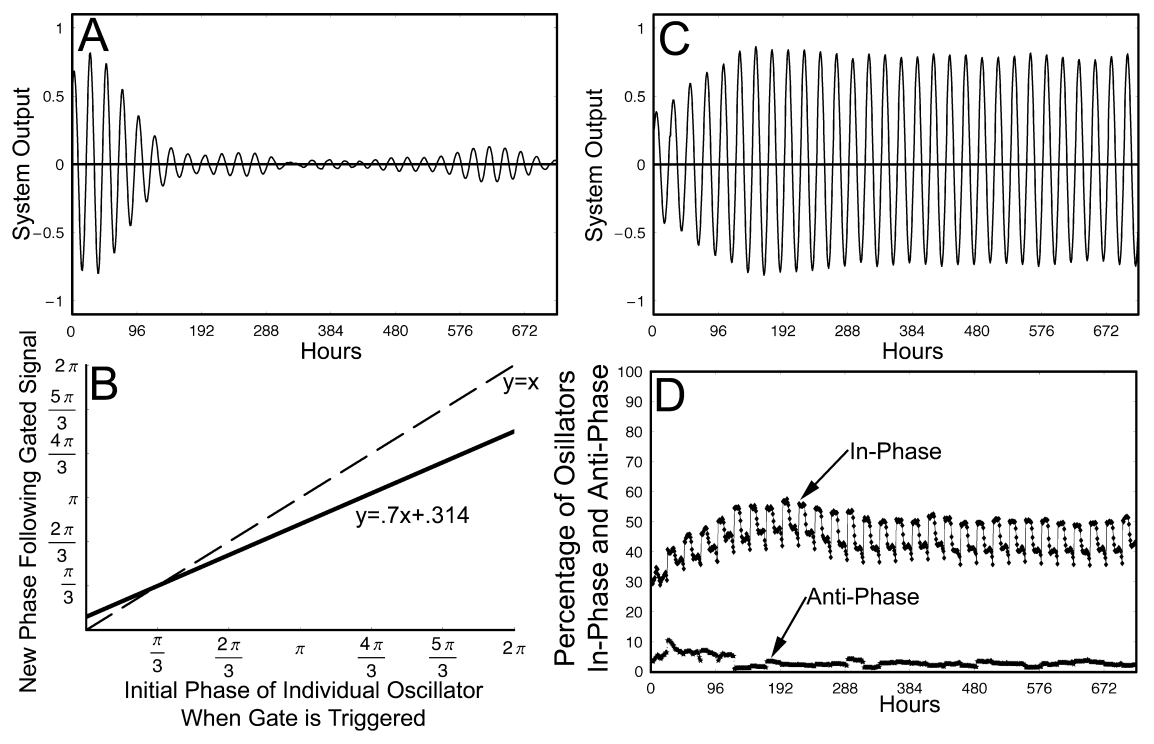

Figure 7. Original gate-oscillator model using a linear phase-resetting function. $\boldsymbol{A}$, The output of an ensemble of oscillators with a mean period of $24 \mathrm{~h}, \mathrm{SD}$ of period of 1.5, and the initial phase with uniform distributions over the following intervals is shown: $60 \%[0, \pi / 3], 5 \%[\pi, 4 \pi / 3], 35 \%[\pi / 3, \pi]$ and $[4 \pi / 3,2 \pi]$, but no gate function. This illustrates how the output of the ensemble decays to near 0 without the influence of the gate. $\boldsymbol{B}$, Phase-resetting function that describes how oscillators are reset by the gate from 0 to $2 \pi$; the dashed line represents no change in-phase. The function has a slope of 0.7 and an intersect of $\pi / 3$. $\boldsymbol{C}$ The output of the ensemble, using the phase-resetting function in $\boldsymbol{B}$, covers $720 \mathrm{~h}$. The threshold for endogenous gate activation was 0.1 , and the oscillators had the same parameters as in $\boldsymbol{A}$. $\boldsymbol{D}$, The percentage of oscillators in-phase and antiphase for the same simulation and time period as $C$; oscillators that are $\pm 2 \mathrm{~h}$ from the mean phase are considered to be in-phase, and oscillators that are $\pm 2 \mathrm{~h}$ from the mean phase offset $12 \mathrm{~h}$ are considered to be in antiphase.

As in the previous model, $r$ represents the radius; $\theta$, the phase angle; $\varepsilon$ controls the weight of the nonlinear component; and $\omega$, the frequency of the oscillator. The scaling factor from the original equation was not incorporated, because it is left at 1 throughout. For these simulations, $\varepsilon$ was set at 0.2 , and $\omega$ was randomly determined based on a normal distribution with a mean period of $24 \mathrm{~h}$ and $\mathrm{SD}$ of $1.5 \mathrm{~h}$. The output of each oscillator is measured as follows: Output $\mathrm{Osc}[i, t]=r_{\mathrm{Osc}[i, t]} \times \cos \left(\theta_{\mathrm{Osc}[i, t]}\right)$. The current simulations were run on ensembles of 500 oscillators, initialized in three subpopulations that are consistent with known properties of SCN neurons (Quintero et al., 2003; Yamaguchi et al., 2003) as follows: the phases of $60 \%$ of the oscillators are uniformly distributed between 0 and $4 \mathrm{~h}$ (which we tentatively identify with oscillators in the shell-like SCN), the phases of $5 \%$ of the oscillators are uniformly distributed between 12 and $16 \mathrm{~h}$, and the rest of the phases of the oscillators are uniformly distributed over the remaining $16 \mathrm{~h}$. The number of oscillators in-phase and antiphase is determined directly from the $\theta$ of the oscillator in each time step. Oscillators $\pm 2 \mathrm{~h}( \pm \pi / 6)$ from the mean phase are said to be in-phase, whereas those $\pm 2 \mathrm{~h}$ from $12 \mathrm{~h}$ away from the mean phase are said to be antiphase.

The gate is essential to maintaining self-sustained output. Activation of the gate provides a daily organizing signal that maintains phase coherence among oscillators and produces selfsustained rhythmic output (Fig. 7A-C) (Antle et al., 2003). This model demonstrates that a gate using a linear phase-resetting function results in a large proportion of oscillators with a common phase (the in-phase population) and a small proportion of oscillators in the opposite phase (the antiphase population) (Fig. $7 D$ ). The individual oscillators in the antiphase population with the linear gate-resetting function, however, change over time in contrast to the localized antiphase population of oscillating neurons identified in vivo (Obrietan et al., 1998; King et al., 2003; Lee et al., 2003a). To better conform to physiological data showing a stable population of antiphase oscillators, in the present model we replace the linear gate phaseresetting function with a sigmoid gate phase-resetting function as follows:

$$
\phi(\theta)=\frac{a}{1+e^{-b \times \theta+c}}+m \times \theta+n .
$$

For the parameter range used in this model, $a$ controls the height of the sigmoid; $b$, how sharply the sigmoid rises; $c$, the $\theta$ of the intersect between the sigmoid and null resetting function; $m$, the skew of the sigmoid; and $n$, the intersect of the sigmoid and the $y$-axis (Fig. 8). This sigmoid phase-resetting function has two attractors and is stable for extreme phases.

To explore the relation between inphase and antiphase cells in the shell- and core-like areas of the SCN, we ran simulations in which the oscillators are initially divided into three subpopulations as described above. We examined several outputs of the simulations: the percentage of oscillators in-phase and antiphase, and whether the same oscillators make up the antiphase population throughout the simulations. Because the antiphase population in split conditions in vivo is concentrated locally, and one of the limitations of the model is that it does not consider spatial distribution of oscillators in the SCN, it is necessary that the oscillators forming the antiphase population in the model remain there for the duration of the simulation.

While refining the model to account for splitting behavior, we retained constraints imposed in the previous model (Antle et al. 2003) and moved from a linear to a sigmoid gate-resetting function that both extends the ability of the model to replicate known behavior and also significantly constrains the applicable parameter set for future development of the model. In the simulation using the linear gate phase-resetting function (Fig. $7 B-D$ ), the mean antiphase population was $3.3 \%$, whereas the mean inphase population was $45.0 \%$. Of the 18 individual oscillators that were in antiphase when the gate fired at hour 172, only 6 of them were still in antiphase when the gate fired at hour 666, which is a correlation of 0.33 . In the simulation using a sigmoid phaseresetting function (Fig. $8 A, B$ ), the mean antiphase population was $5.6 \%$, and the mean in-phase population was $61.9 \%$. Of the 31 individual oscillators that were in antiphase when the gated fired at hour 171, 24 of them were still in antiphase when the gated fired at hour 661, which is a correlation of 0.77 . When the sigmoid phase-resetting function was moved to the left (Fig. $8 A, C$ ), by shifting the parameter $c$ in Equation 2 from -4 to -3 , the resulting simulation had a mean antiphase population of $15.7 \%$ and a mean in-phase population of $51.7 \%$. Of the 100 individual oscillators that were in antiphase when the gate fired at hour 163, 90 of them were still in antiphase when the gate fired at hour 674, which is a correlation of 0.90 (Fig. $8 C, D, G)$. The hours chosen are based on when the gate fired; as the gate fires because of the endogenous output, this ensures consistent circadian time. 
Additional simulations using the same general parameters replicated the data of the simulations discussed above and shown in Figures 7 and 8.

\section{Discussion}

By analyzing the distinct responses of two subregions of the SCN in split animals, the present study reveals novel aspects of pacemaker organization. First, in the split animal, two populations of circadian oscillators in each SCN cycle in antiphase. Second, PER1 ICC indicates that, within each SCN, the fundamental change in the split state occurs in the network of the core-like area. Importantly, the population of cells that cycle coherently in antiphase in the core-like SCN is much larger in split animals than in controls. Finally, rhythmic expression of hypothalamic targets of SCN efferents is in-phase with the ipsilateral core-like region for some sites and with the ipsilateral shell-like region for others.

\section{Significance of antiphase rhythms in core- and shell-like SCN}

A key result in the present experiments is the discovery within each SCN of a coherent rhythmic oscillation in the core-like SCN that cycles in antiphase to the main population lying in the shell-like region of the nucleus. The primary difference between split and unsplit animals lies in the coherent rhythm of cells lying in the corelike region. Neurons in the SCN are heterogeneous, and cells in the core have important properties, quite distinct from those in the shell-like SCN (Antle and Silver, 2005). Microlesions of the CalBcontaining SCN region of hamsters eliminate circadian rhythms of locomotor activity, drinking, gnawing, body temperature, and hormone secretion, although substantial SCN tissue survives ablation

(LeSauter and Silver, 1999; Kriegsfeld et al., 2004b). CalBexpressing cells in the SCN are directly retinorecipient (Bryant et al., 2000) and express light-induced c-FOS (Silver et al., 1996a), Per1, and Per2, but lack detectable rhythms in these genes under LD and DD conditions (Hamada et al., 2001). The present results extend this work by showing constant low PER1 protein expression in the core-like region of unsplit control animals housed in DD or LL. Finally, we established that rhythmicity does not occur in previously characterized nonoscillating, Calb-containing cells, but the identity of the coherently rhythmic antiphase cells in the core-like region remains to be determined.

The novel result of the present study, high-amplitude antiphase rhythmic expression of PER1 protein rhythm in the corelike SCN, was not recognized previously (de la Iglesia et al., 2000; Ohta et al., 2005). This may be explained by the fact that markers for specific SCN regions were not available when the split hamster SCN was initially described (de la Iglesia et al., 2000). With regard to the behaviorally split mouse, the explanted SCN model used by
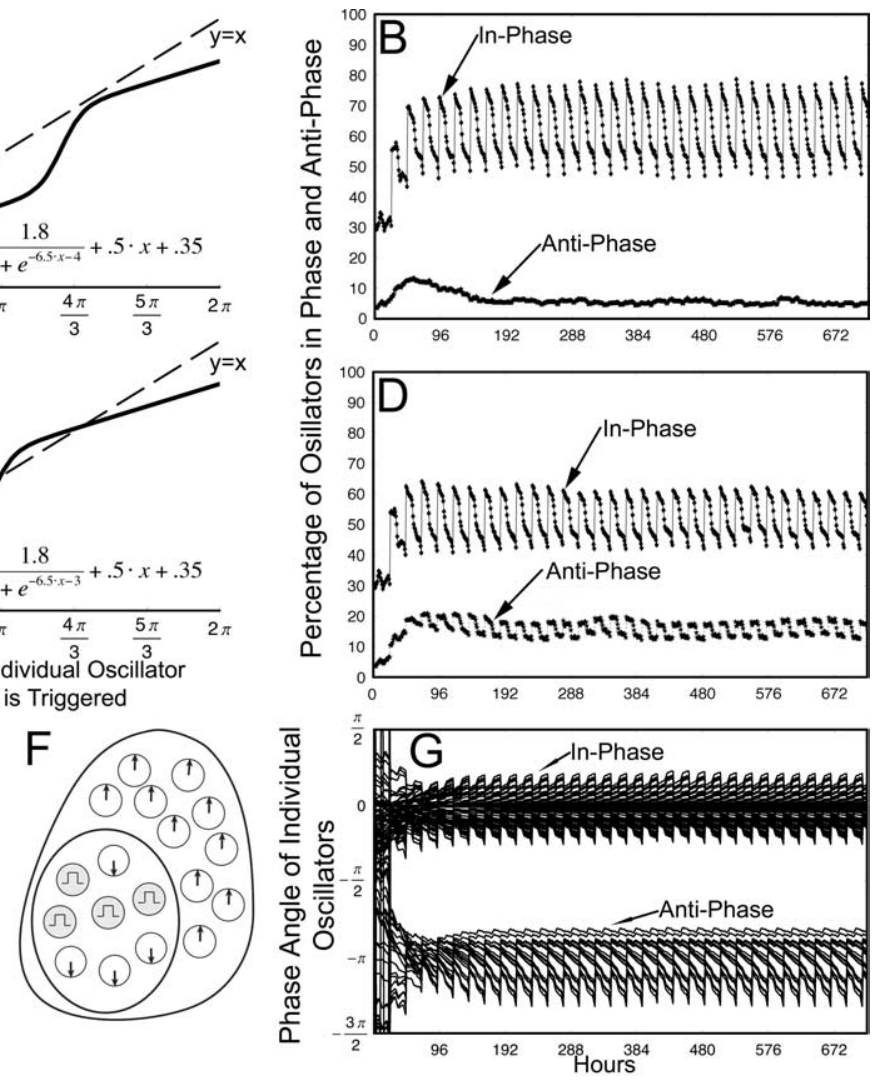

Figure 8. Gate-oscillator model using a sigmoid phase-resetting function. $\boldsymbol{A}$, Phase-resetting function that describes how oscillators are reset for the simulation shown in $\boldsymbol{B}$; the dashed line represents no change in phase. $\boldsymbol{B}$, The percentage of oscillators in-phase and antiphase for a simulation of $720 \mathrm{~h}$ using the phase-resetting function in $\boldsymbol{A}$; 0scillators that are $\pm 2 \mathrm{~h}$ from the mean the following intervals: $60 \%[0, \pi / 3], 5 \%[\pi, 4 \pi / 3], 35 \%[\pi / 3, \pi]$ and $[4 \pi / 3,2 \pi]$. C, Phase-resetting function that of determinations were made using the same criteria as in $\boldsymbol{B}$; likewise, oscillators had the same parameters as in $\boldsymbol{B}$. $\boldsymbol{E}$, Schematic of an SCN in control conditions; the shell oscillators are in-phase, a small population of the core-like oscillators are antiphase, and the remaining core-like oscillators are not cohesive. The gate is represented by the gray cells, and the direction of the arrows represent of $30 \%$ of the individual oscillators that were in-phase or antiphase at both the beginning and end of the simulation shown in $\boldsymbol{D}$. This is representative of the entire population of stable in-phase and stable antiphase oscillators and demonstrates that individual oscillators do not wander from one community to the other.

Ohta and colleagues (2005) necessarily lacks retinal input, eliminating the possibility of finding a retina-dependent antiphase rhythm (Lee et al., 2003a).

\section{Modeling photic effects}

The model can account for a key physiological finding in this paper: the increase in the proportion of antiphase oscillators in split hamsters. The coherent antiphase rhythm in the core-like region emerges after prolonged exposure to light. It is possible that such light exposure induces rhythmicity in neurons that were previously arrhythmic. However, our model favors the alternate hypothesis that light produces coherence in the phase of core-like SCN oscillators lying scattered among CalB gate cells. Physiologically, the retinorecipient nonrhythmic CalB cells are the gates, whereas the remaining nearby $\mathrm{SCN}$ cells are oscillators (Fig. 8E,F). The resetting signals from the gate cells are essential to maintain the phase coherence among individual oscillators under constant conditions. In the present model, the impact on 
gate function of the change from DD to LL is represented by a relatively small change in a single parameter, $c$ (Eq. 2), of the sigmoid phase-resetting function and leads to qualitative and quantitative changes in the relative proportion of in-phase and antiphase oscillators similar to those seen in physiological studies. This suggests a physiological change in the regulation of the retinorecipient gate cells between DD and LL. This phenomenon is indicated by the emerging phase coherence of previously unorganized oscillator cells into the coherent antiphase population. In summary, the model suggests the testable hypothesis that the effect of light is to change the behavior of the retinorecipient gate cells, and/or gate-oscillator interactions so as to produce synchronization among individual oscillators in the core-like region of each split SCN. This model does not, however, explicitly represent the two symmetrical lobes of the nucleus.

\section{Implications of the results for understanding evening and morning oscillator model}

It has long been thought that the circadian system consists of two coupled equivalent, but functionally distinct oscillators termed "morning (M) oscillator" and "evening (E) oscillator" (Pittendrigh and Daan, 1976; Daan and Berde, 1978). Several attractive hypotheses have been made regarding the localization and mechanism of $\mathrm{M}$ and $\mathrm{E}$ oscillators. One such suggestion involves antiphase oscillations of clock genes in the left and right SCN of the split hamster (de la Iglesia et al., 2000). It has also been proposed that $\mathrm{M}$ and $\mathrm{E}$ oscillators are organized at the level of genes, with Per1/Cry1 being the $\mathrm{M}$ and Per2/Cry2 being the E (Daan et al., 2001), although this view was challenged in light of evidence that the protein product of these genes are virtually in-phase (Hastings, 2001). Other evidence indicates two oscillator populations within each SCN. Thus, two peaks of electrical activity, one locked to dawn and the other locked to dusk, were observed in horizontal SCN slices indicating that each SCN has two oscillating components (Jagota et al., 2000). The present study suggests the possibility that oscillators of the core and shell SCN constitute two distinct networks of topographically separate E and M oscillators. Spatially separate $\mathrm{M}$ and $\mathrm{E}$ oscillators have been described in flies bearing mutations and genetic manipulation in specific cells (Grima et al., 2004; Stoleru et al., 2004). The population of antiphase oscillators within each SCN could also provide the basis for the internal coincidence of two oscillator populations long postulated to underlie the changes in photosensitivity underlying seasonality (Pittendrigh, 1972).

\section{Each oscillating SCN compartment is associated with activated target sites in the brain}

A fundamental question regarding SCN coupling is how the activity of oscillators produces coherent rhythms in SCN target sites. Our results from examination of the temporal relationship between c-FOS expression in distinct SCN areas, and in adjacent hypothalamic regions in split animals, indicates that unilateral activation can occur in at least two distinct hypothalamic regions, the PVN and the LSPVZ. Activation of PVN was observed on the side ipsilateral to the activated core-like region, whereas activation of LSPVZ was found on the side ipsilateral to activated shelllike SCN. This is consistent with anatomical tract tracing indicating that core and shell SCN regions both contain projection neurons (Kriegsfeld et al., 2004a). Previous studies indicate that locomotor activity can be regulated by a diffusible SCN output signal (Silver et al., 1996b), whereas neurosecretory neurons require ipsilateral point-to-point axonal connections (Lehman et al., 1987; Meyer-Bernstein et al., 1999; de la Iglesia et al., 2003).
Together, the data strongly support the existence of a multiplicity of SCN output mechanisms.

\section{Broader implications}

The appearance of a high-amplitude rhythm in the retinorecipient core of the SCN is a major finding in the present study and draws attention to the effects of prolonged light exposure. It has been shown that a photic stimulus provides drive on the human circadian pacemaker, assessed by melatonin secretion, and this effect is related to previous photic history (Smith et al., 2004). Constant light exposure restores the circadian rhythms of both Per 2 and clock mutant mice, which are arrhythmic in DD (Spoelstra et al., 2002; Steinlechner et al., 2002). Furthermore, mice bearing a deficient Perl gene are not able to adapt to the challenging photoperiods, suggesting that photic drive on the circadian pacemaker may be mediated through the Per1 gene (Masubuchi et al., 2005). Given that the network organization of clock cells in the SCN distinguishes them from oscillators in other tissues (Yamazaki et al., 2000; Welsh et al., 2004; Nagoshi et al., 2005), the modulation of SCN network elements by environmental cues, both tonic and acute, is key to unraveling how the coherent organization and function of the master clock of the brain are achieved.

\section{References}

Abrahamson EE, Moore RY (2001) Suprachiasmatic nucleus in the mouse: retinal innervation, intrinsic organization and efferent projections. Brain Res 916:172-191.

Antle MC, Silver R (2005) Orchestrating time: arrangements of the brain circadian clock. Trends Neurosci 28:145-151.

Antle MC, Foley DK, Foley NC, Silver R (2003) Gates and oscillators: a network model of the brain clock. J Biol Rhythms 18:339-350.

Antle MC, Kriegsfeld LJ, Silver R (2005) Signaling within the master clock of the brain: localized activation of mitogen-activated protein kinase by gastrin-releasing peptide. J Neurosci 25:2447-2454.

Bryant DN, LeSauter J, Silver R, Romero MT (2000) Retinal innervation of calbindin-D28K cells in the hamster suprachiasmatic nucleus: ultrastructural characterization. J Biol Rhythms 15:103-111.

Chambille I, Doyle S, Servière J (1993) Photic induction and circadian expression of Fos-like protein immunohistochemical study in the retina and suprachiasmatic nuclei of hamster. Brain Res 612:138-150.

Daan S, Berde C (1978) Two coupled oscillators: simulations of the circadian pacemaker in mammalian activity rhythms. J Theor Biol 70:297-313.

Daan S, Albrecht U, van der Horst GT, Illnerova H, Roenneberg T, Wehr TA, Schwartz WJ (2001) Assembling a clock for all seasons: are there $\mathrm{M}$ and E oscillators in the genes? J Biol Rhythms 16:105-116.

de la Iglesia HO, Meyer J, Carpino Jr A, Schwartz WJ (2000) Antiphase oscillation of the left and right suprachiasmatic nuclei. Science 290:799-801.

de la Iglesia HO, Meyer J, Schwartz WJ (2003) Lateralization of circadian pacemaker output: activation of left- and right-sided luteinizing hormone-releasing hormone neurons involves a neural rather than a humoral pathway. J Neurosci 23:7412-7414.

Field MD, Maywood ES, O’Brien J, Weaver DR, Reppert S, Hastings MH (2000) Analysis of clock proteins in mouse SCN demonstrates phylogenic divergence of the circadian clockwork and resetting mechanisms. Neuron 25:437-447.

Grima B, Chelot E, Xia R, Rouyer F (2004) Morning and evening peaks of activity rely on different clock neurons of the Drosophila brain. Nature 431:869-873.

Guido ME, Goguen D, De Guido L, Robertson HA, Rusak B (1999) Circadian and photic regulation of immediate-early gene expression in the hamster suprachiasmatic nucleus. Neuroscience 90:555-571.

Hamada T, LeSauter J, Venuti JM, Silver R (2001) Expression of Period genes: rhythmic and nonrhythmic compartments of the suprachiasmatic nucleus pacemaker. J Neurosci 21:7742-7750.

Hastings M (2001) Modeling the molecular calendar. J Biol Rhythms 16:117-123.

Jagota A, de la Iglesia HO, Schwartz WJ (2000) Morning and evening circa- 
dian oscillations in the suprachiasmatic nucleus in vitro. Nat Neurosci 3:372-376.

Jobst EE, Allen CN (2002) Calbindin neurons in the hamster suprachiasmatic nucleus do not exhibit a circadian variation in spontaneous firing rate. Eur J Neurosci 16:2469-2474.

King VM, Chahad-Ehlers S, Shen S, Harmar AJ, Maywood ES, Hastings MH (2003) A hVIPR transgene as a novel tool for the analysis of circadian function in the mouse suprachiasmatic nucleus. Eur J Neurosci 17:822-832.

Klein DC, Moore RY, Reppert SM, eds (1991) Suprachiasmatic nucleus: the mind's clock. New York: Oxford UP.

Kriegsfeld LJ, Leak RK, Yackulic CB, LeSauter J, Silver R (2004a) Organization of suprachiasmatic nucleus projections in Syrian hamsters $(\mathrm{Me}$ socricetus auratus): an anterograde and retrograde analysis. J Comp Neurol 468:361-379.

Kriegsfeld LJ, LeSauter J, Silver R (2004b) Targeted microlesions reveal novel organization of the hamster suprachiasmatic nucleus. J Neurosci 24:2449-2457.

Lee HS, Nelms JL, Nguyen M, Silver R, Lehman MN (2003a) The eye is necessary for a circadian rhythm in the suprachiasmatic nucleus. Nat Neurosci 6:111-112.

Lee HS, Billings HJ, Lehman MN (2003b) The suprachiasmatic nucleus: a clock of multiple components. J Biol Rhythms 18:435-449.

Lehman MN, Silver R, Gladstone WR, Kahn RM, Gibson M, Bittman EL (1987) Circadian rhythmicity restored by neural transplant. Immunocytochemical characterization of the graft and its integration with the host brain. J Neurosci 7:1626-1638.

LeSauter J, Silver R (1999) Localization of a suprachiasmatic nucleus subregion regulating locomotor rhythmicity. J Neurosci 19:5574-5585.

Masubuchi S, Kataoka N, Sassone-Corsi P, Okamura H (2005) Mouse Period1 (mPER1) acts as a circadian adaptor to entrain the oscillator to environmental light/dark cycles by regulating mPER2 protein. J Neurosci 25:4719-4724.

Meyer-Bernstein EL, Jetton AE, Matsumoto SI, Markuns JF, Lehman MN, Bittman EL (1999) Effects of suprachiasmatic transplants on circadian rhythms of neuroendocrine function in golden hamsters. Endocrinology 140:207-218

Moore RY (1973) Retinohypothalamic projection in mammals: a comparative study. Brain Res 49:403-409.

Moore RY (1996) Entrainment pathways and the functional organization of the circadian system. Prog Brain Res 111:103-119.

Nagoshi E, Brown SA, Dibner C, Kornmann B, Schibler U (2005) Circadian gene expression in cultured cells. Methods Enzymol 393:543-557.

Nuesslein-Hildesheim B, O’Brien JA, Ebling FJ, Maywood ES, Hastings MH (2000) The circadian cycle of mPER clock gene products in the suprachiasmatic nucleus of the Siberian hamster encodes both daily and seasonal time. Eur J Neurosci 12:2856-2864.

Obrietan K, Impey S, Storm DR (1998) Light and circadian rhythmicity regulate MAP kinase activation in the suprachiasmatic nuclei. Nat Neurosci 1:693-700.

Ohta H, Yamazaki S, McMahon DG (2005) Constant light desynchronizes mammalian clock neurons. Nat Neurosci 8:267-269.

Pittendrigh CS (1972) Circadian surfaces and the diversity of possible roles of circadian organization in photoperiodic induction. Proc Natl Acad Sci USA 69:2734-2737.

Pittendrigh CS, Daan S (1976) A functional analysis of circadian pacemak- ers in nocturnal rodents V. Pacemaker structure: a clock for all seasons. J Comp Physiol 106:291-331.

Quintero JE, Kuhlman SJ, McMahon DG (2003) The biological clock nucleus: a multiphasic oscillator network regulated by light. J Neurosci 23:8070-8076.

Reppert SM, Weaver DR (2001) Molecular analysis of mammalian circadian rhythms. Annu Rev Physiol 63:647-676.

Shimomura K, Kornhauser JM, Wisor JP, Umezu T, Yamazaki S, Ihara NL, Takahashi JS, Menaker M (1997) Circadian behavior and plasticity of light-induced c-fos expression in SCN of tau mutant hamsters. J Biol Rhythms 13:305-314.

Silver R, Romero MT, Besmer HR, Leak R, Nunez JM, LeSauter J (1996a) Calbindin-D28K cells in the hamster SCN express light-induced Fos. NeuroReport 7:1224-1228.

Silver R, LeSauter J, Tresco PA, Lehman MN (1996b) A diffusible coupling signal from the transplanted suprachiasmatic nucleus controlling circadian locomotor rhythms. Nature 382:810-813.

Smith KA, Schoen MW, Czeisler CA (2004) Adaptation of human pineal melatonin suppression by recent photic history. J Clin Endocrinol Metab 89:3610-3614.

Spoelstra K, Oklejewicz M, Daan S (2002) Restoration of self-sustained circadian rhythmicity by the mutant clock allele in mice in constant illumination. J Biol Rhythms 17:520-525.

Steinlechner S, Jacobmeier B, Scherbarth F, Dernbach H, Kruse F, Albrecht U (2002) Robust circadian rhythmicity of Per1 and Per2 mutant mice in constant light, and dynamics of Per1 and Per2 gene expression under long and short photoperiods. J Biol Rhythms 17:202-209.

Stoleru D, Peng Y, Agosto J, Rosbash M (2004) Coupled oscillator control morning and evening locomotor behaviour of Drosophila. Nature 431:862-868.

Sudo M, Sasahara K, Moriya T, Akiyama M, Hamada T, Shibata S (2003) Constant light housing attenuates circadian rhythms of mPer2 mRNA and MPER2 protein expression in the suprachiasmatic nucleus of mice. Neuroscience 121:493-499.

Sumova A, Sladek M, Jac M, Illnerova H (2002) The circadian rhythm of Perl gene product in the rat suprachiasmatic nucleus and its modulation by seasonal changes in daylength. Brain Res 947:260-270.

van den Pol AN, Tsujimoto KL (1985) Neurotransmitters of the hypothalamic suprachiasmatic nucleus: immunocytochemical analysis of 25 neuronal antigens. Neuroscience 15:1049-1086.

Welsh DK, Logothetis DE, Meister M, Reppert SM (1995) Individual neurons dissociated from rat suprachiasmatic nucleus express independently phased circadian firing rhythms. Neuron 14:697-706.

Welsh DK, Yoo SH, Liu AC, Takahashi JS, Kay SA (2004) Bioluminescence imaging of individual fibroblasts reveals persistent, independently phased circadian rhythms of clock gene expression. Curr Biol 14:2289-2295.

Yamaguchi S, Isejima H, Matsuo T, Okura R, Yagita K, Kobayashi M, Okamura H (2003) Synchronization of cellular clocks in the suprachiasmatic nucleus. Science 302:1408-1412.

Yamazaki S, Numano R, Abe M, Hida A, Takahashi R, Ueda M, Block GD, Sakaki Y, Menaker M, Tei H (2000) Resetting central and peripheral circadian oscillators in transgenic rats. Science 288:682-685.

Yan L, Silver R (2004) Resetting the brain clock: time course and localization of $\mathrm{mPER} 1$ and $\mathrm{mPER} 2$ protein expression in suprachiasmatic nuclei during phase shifts. Eur J Neurosci 19:1105-1109. 